\title{
Pathogenesis of Dengue viral infections
}

\author{
Gathsaurie Neelika Malavige, ${ }^{1,2}$ Neluka Fernando, ${ }^{1}$ Graham Ogg ${ }^{2,3}$ \\ Sri Lankan Journal of Infectious Diseases2011; Vol.1(1): 2-8
}

DOI: http://dx.doi.org/10.4038/sljid.v1i1.2987

Key words : Dengue; immunopathology; T cells; antibodies; viraemia

\begin{abstract}
Dengue viral infections are one of the most important mosquito borne viral infections in the world. There are four dengue virus serotypes (DEN1-4) which are closely related. Initial infection with a particular serotype is known as primary infection, which is usually asymptomatic or results in mild disease manifestations. Although cross reactive $\mathrm{T}$ cells and cross reactive antibodies have been shown to contribute to disease pathogenesis, these mechanisms alone do not explain the immunopathological mechanisms leading to severe disease.However, studies have suggested that immunopathological, host genetic and viral factors all contribute to the occurrence of severe disease. Therefore, it is important to further investigate the quality of dengue specific immune responses in patients with acute severe dengue and asymptomatic dengue infection. This would help determine the immune correlates of severe clinical disease and protection.
\end{abstract}

\section{Introduction}

Dengue viral infections are one of the most important mosquito borne viral infections in the world. In the past fifty years, its incidence has increased 30 -fold with significant outbreaks occurring in five of six WHO regions. It is estimated that 2.1 million cases of dengue haemorrhagic fever (DHF)/dengue shock syndrome (DSS) occur every year resulting in 21,000 deaths. ${ }^{1}$

\section{Dengue infections in Sri Lanka}

Sri Lanka has been affected by epidemics of DHF for over 2 decades. Dengue viral infections have been endemic in Sri Lanka since the mid 1960s which was when the first cases of DF/DHF were reported. ${ }^{2,3}$ Although the Sri Lankan population had been exposed to the virus for decades, severe forms of dengue infection (DHF/DSS) was rare. However, in recent years dengue has become the number one killer mosquito borne infection in Sri Lanka. ${ }^{4}$ Sri Lanka experienced the worst ever dengue outbreak in 2009 and 2010 with 35000 cases in 2009 with 346 deaths and 32292 cases and 229 deaths in the 2010. Not only is the incidence of dengue infections rising in Sri Lanka, but also the infection is spreading to all parts of Sri Lanka.

\footnotetext{
${ }_{2}^{1}$ Department of M icrobiology, Faculty of M edical Sciences, University of Sri Jayawardanapura, Sri Lanka.

${ }_{2}^{2}$ M RC Human Immunology Unit, Weatherall Institute of M olecular M edicine, Oxford

${ }^{3}$ Department of Dermatology, Churchill Hospital, Oxford, OX3 7LJ, UK

Address for correspondence : Neelika Malavige, Department of Microbiology, Faculty of Medical

Sciences, University of Sri Jayawardanapura, Sri Lankaｅmail:gathsaurie.malavige@ndm.ox.ac.uk
} 


\section{Distribution of suspected DF/DHF by week, Sri Lanka 2004 -2010 (up to 07/01/2011)}

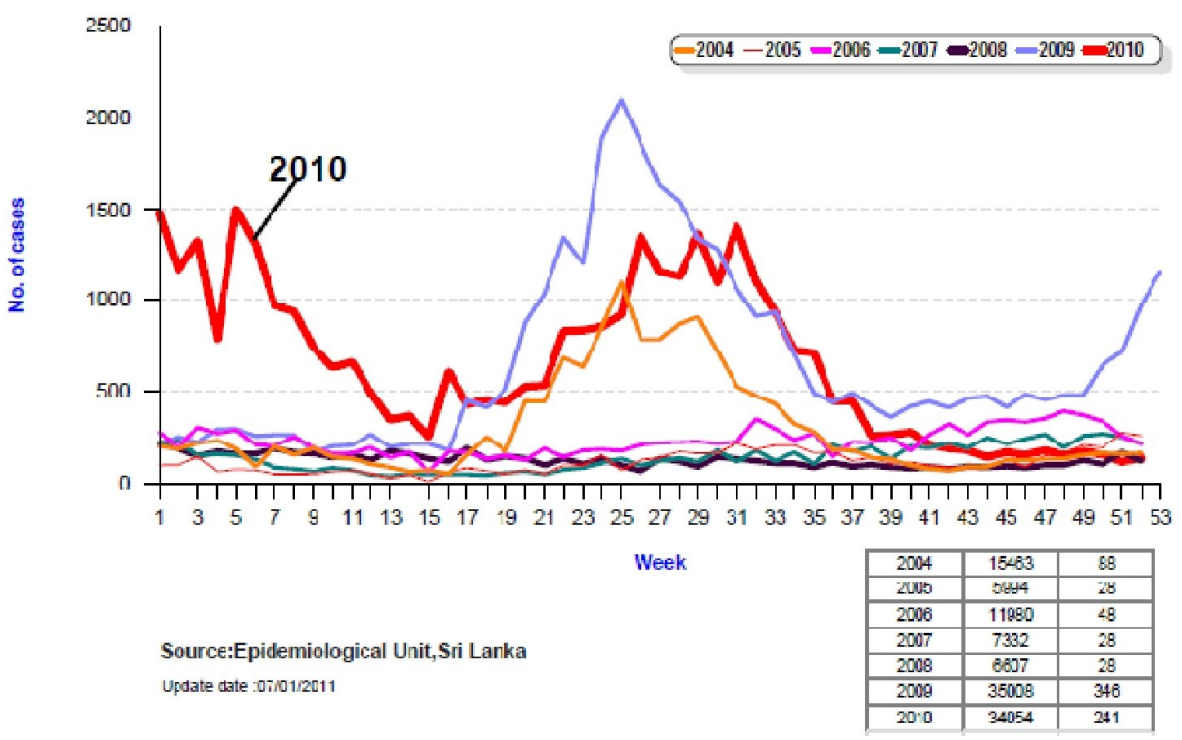

Fig 1: Dengue fever/DHF cases reported to the Sri Lanka Epidemiology unit from 2004 to 2010

Although the reasons for this massive dengue epidemic in recent years are not clear, PCR and virus isolation studies carried out by our laboratory and Genetech Research institute (Dr. Dharshan De Silva, personal communication) show that the displacement of the predominant circulating dengue virus serotype is an important contributing factor. ${ }^{5}$ Dengue virus serotypes 2 and 3 were the predominant circulating serotypes until 2009 and were responsible for $86 \%$ of dengue infections. Dengue 1 serotype only accounted for $7 \%$ of infections ${ }^{6}$. PCR results of patients with DHF recruited from August 2010 to December 2010 showed that dengue 1 serotype accounted for more than $95 \%$ of dengue infections, ${ }^{5}$ which was similar to what was observed by the Sri Lanka epidemiology unit (Dr. Hasitha Tissera, Sri Lanka epidemiology unit, personal communication). Therefore, this serotype shift is probably contributed in some way to the massive dengue outbreak that occurred in the last 2 years.

\section{Dengue pathogenesis}

The four dengue virus serotypes (DEN1-4) which cause dengue are closely related and have a $65-70 \%$ sequence homology. Initial infection with a particular serotype is known as primary infection, which is usually asymptomatic or results in mild disease manifestations. ${ }^{8}$ Subsequent infection with other serotypes (secondary dengue infections) may lead to severe disease which manifests in the form of DHF/DSS. ${ }^{8}$ However, only $0.18-1 \%$ of those with primary infections and $2-9 \%$ of secondary infections manifest as DHF/DSS. ${ }^{8}$ Therefore, the majority of individuals who are infected with the dengue virus do not develop symptomatic disease or only develop mild clinical manifestations. The occurrence of DHF/DSS is thought to result from a complex interplay between the virus, host genetic makeup and host immune 
factors. The pathophysiology of dengue viral infections and factors that result in severe clinical disease is poorly understood.

\section{Dengue virus serotype and disease pathogenesis}

All dengue virus serotypes have been associated with severe clinical disease. However, certain genotypes of dengue virus serotypes 2 and 3 have been shown to be more virulent and the cause of massive dengue epidemics. ${ }^{6,9}$ For instance, some studies have suggested that the emergence of DHF in Sri Lanka in 1989 coincided with the appearance of a new DEN-3, subtype III variant. ${ }^{10}$ Dengue virus serotype 4 has been shown to cause milder clinical disease. However, dengue serotype 1 has been associated with more severe disease during primary dengue infections. ${ }^{11}$ In fact, it was recently shown that patients with primary infection with dengue 1 serotype had higher viral loads than those infected with other viral serotypes. ${ }^{12}$

\section{Genetic factors that contribute to severe clinical disease}

Several genetic factors have been shown to be associated with the development of DHF/DSS and some have been shown to be protective. ${ }^{13-15}$ Certain HLA- class I and class II alleles, ${ }^{13,14,16,17}$ polymorphisms in the tumor necrosis factor alpha(TNF- $\alpha$ ), Vitamin D receptor, ${ }^{18}$ CTLA-4 and transforming growth factor $\beta$ (TGF- $\left.\beta\right)^{19}$ have been shown to be associated with development of DHF/DSS. ${ }^{15}$ We have conducted studies to identify possible HLA- class I and class II alleles, which increase the risk of developing DHF/DSS during primary and secondary dengue in the Sri Lankan population. We found that HLA-A*31 and DRB $1 * 08$ were significantly associated with susceptibility to DSS when infected with the dengue virus, during secondary dengue infection. The frequency of DRB1*08 allele was 28.7 times higher than in the normal population in patients with DSS. HLA-A*31 allele was increased 16.6 fold in DHF who developed shock when compared to those who did not develop shock. HLA-A*24 and DRB1*12 were significantly associated with the development of DHF during primary dengue infection. ${ }^{20}$ Therefore, such HLA allele associations with clinical disease severity suggest that certain HLA alleles confer susceptibility/ protection to severe dengue infections.

\section{Host immune factors responsible for severe disease: cross reactive antibodies}

It has been shown that cross reactive $\mathrm{T}$ cells and antibodies are thought to contribute to disease pathogenesis in secondary dengue infections. Poorly neutralizing and disease enhancing antibodies are thought to cause disease pathogenesis by increasing infection of cells such as macrophages and dendritic cells. ${ }^{15}$ It is thought that proinflammatory cytokines which result in endothelial dysfunction are secreted in large quantities as more and more cells are infected. High levels of enhancing antibodies are also thought to be the cause of the high frequency of DHF during primary infection during infants. ${ }^{21}$ However, this 'antibody enhancement hypothesis' has been questioned by many, since several studies have shown that there was no difference in the viral loads in primary and secondary dengue infection. ${ }^{22-24}$ Although, viral loads have not been found to be higher in patients with secondary dengue, higher viral loads are thought to correlate with clinical disease severity as viral loads are higher in patients with DHF when compared to those with DF. Furthermore, it has been shown that individuals with severe clinical disease have prolonged viraemia than those with milder disease. ${ }^{23}$ It therefore appears that severe dengue is associated with an inability of the immune system to eliminate the virus. 


\section{Cross reactive $\mathbf{T}$ cells}

Massive immune activation of T cells, ${ }^{25-27}$ monocytes ${ }^{28}$ and macrophages have been shown to produce unfavourable cytokines such as $\mathrm{TNF} \alpha$ in large quantities. Cross reactive memory $\mathrm{T}$ cells have been shown to contribute to immunopathology by altering the cytokine profiles during secondary infection and are also believed to be less effective in eliminating the newly infecting virus serotype ${ }^{29,30}$ which is thought to lead to enhanced viral replication and thus severe clinical disease.

$\mathrm{T}$ cell responses of individuals who are naturally infected with the virus were shown to be directed at all proteins of the DV, and specifically the NS3 region. ${ }^{27,31} \mathrm{~T}$ cell responses generated during acute infection have been shown to be highly cross reactive. However, their cytokine secretion and cytolytic functions depended on the nature of the interactions of the DV antigen, MHC and $\mathrm{T}$ cell receptor complex and subsequent signaling events. Therefore, it has been suggested that $\mathrm{T}$ cell responses to the newly infecting DV serotype depend on the existing DV specific memory $\mathrm{T}$ cell repertoire. ${ }^{32}$ Imrie et al have shown that even single substitutions of the amino acid sequence of the DV $\mathrm{T}$ cell epitope altered the cytokine secretion patterns such as TNF $\alpha$, IFN $\gamma$ and IL-4. ${ }^{32}$ Cross reactive DV-specific T cells have been shown to have suboptimal degranulation capacity, but secrete high levels of cytokines. ${ }^{30}$ Reduced or suboptimal antiviral responses are therefore also thought to contribute to disease pathogenesis in acute dengue infection. ${ }^{29}$

Severe clinical disease, which has even resulted in fatalities is known to occur during primary dengue viral infections in the absence of cross reactive antibodies and DV-specific T cells. ${ }^{33,34}$ Primary dengue infection resulting in DHF has been shown to be commoner among infants ${ }^{21}$ and in pregnant women. ${ }^{35}$ In addition, studies carried out by us in individuals with previous asymptomatic/mild infection have shown that highly cross reactive dengue specific memory $\mathrm{T}$ cell are also seen in these individuals ${ }^{36}$. Therefore, it appears that the presence of cross reactive $\mathrm{T}$ cells and antibodies alone do not contribute to the development of severe dengue infections.

As it is evident that factors other than cross reactive $\mathrm{T}$ cells and antibodies are likely to play a role in the disease pathogenesis, some groups have investigated the role of immune regulatory mechanisms in acute dengue infection. CD4+ Foxp3 expressing regulatory T cells (Tregs) are the main regulators of the adaptive immune system. Some studies have suggested that a defective immune suppression or a defect in regulatory $\mathrm{T}$ cells could contribute to severe clinical disease. For instance, a high regulatory T cell (Tregs)/ effector T cell ratio has been shown to be associated with milder clinical disease ${ }^{37}$. This suggests that patients with severe clinical disease possibly have impaired functional regulatory T cells responses. However IL10 , which is the main regulatory $\mathrm{T}$ cell cytokine, has been found to be significantly higher in patients with severe disease when compared to those with milder clinical symptoms. ${ }^{38}$

Collectively, all this evidence suggests that the pathogenesis of DHF is far more complex than previously thought. Therefore, more detailed studies in patients with acute severe dengue infection and asymptomatic infection should be carried out in order to try and understand this complex immunopathogenesis. 


\section{Summary}

Dengue viral infections are an important cause of mortality and morbidity in Sri Lanka and the rest of the world. Dengue virus factors, host genetic and immunological factors are thought to act together and result in severe clinical disease. Although the pathogenesis of dengue infections have been widely studied, the disease mechanisms are still poorly understood. It is therefore important to further investigate the quality of dengue specific immune responses in patients with acute severe and asymptomatic dengue infection, to determine the immune correlates of severe clinical disease and protection.

\section{References}

1. Callaway E. Dengue fever climbs the social ladder. Nature 2007; 448: 734-735.

2. Munasinghe DR, Amarasekera PJ and Fernando CF. An epidemic of dengue-like fever in Ceylon (chikungunya--a clinical and haematological study. Ceylon Medical Journal 1966; 11: 129-42.

3. Mendis NM. Epidemiology of dengue-like fever in Ceylon. Ceylon Medical Journal 1967; 12: 67-74.

4. Gagnon SJ, Mori M, Kurane I, et al. Cytokine gene expression and protein production in peripheral blood mononuclear cells of children with acute dengue virus infections. Journal of Medical Virology 2002; 67: 41-6.

5. Atukorale V, Meedin, F., Malavige, G.N., Wijesinghe, T, Jayaratne, S.D., Fernando, N., Ogg, G.S.,. Molecular characteristics of dengue viral serotypes responsible for dengue epidemic in year 2010.In: Sri Lanka Medical Association Annual Congress. Colombo, 2011

6. Kanakaratne N, Wahala WM, Messer WB, et al. Severe dengue epidemics in Sri Lanka, 2003-2006. Emerging Infectious Diseases 2009; 15: 192-9.

7. Weaver SC, Vasilakis N. Molecular evolution of dengue viruses: contributions of phylogenetics to understanding the history and epidemiology of the preeminent arboviral disease. Infection Genetics and Evolution 2009; 9: 523-40.

8. Mathew A, Rothman AL. Understanding the contribution of cellular immunity to dengue disease pathogenesis. Immunological Reviews 2008; 225: 300-13.

9. Guzman MG, Kouri G, Valdes L, Bravo J, Vazquez S and Halstead SB. Enhanced severity of secondary dengue-2 infections: death rates in 1981 and 1997 Cuban outbreaks. Revista Panamericana de Salud Pública2002; 11: 223-7.

10. Messer WB, Gubler DJ, Harris E, Sivananthan K and de Silva A M. Emergence and global spread of a dengue serotype 3, subtype III virus. Emerging Infectious Diseases 2003; 9: 800-9.

11. Kyle JL, Harris E. Global spread and persistence of dengue. Annual Review of Microbiology 2008; 62: 71-92.

12. Duyen HT, Ngoc TV, Ha DT, et al. Kinetics of Plasma Viremia and Soluble Nonstructural Protein 1 Concentrations in Dengue: Differential Effects According to Serotype and Immune Status. Journal of Infectious Diseases 2011; 203:1292- 300.

13. Nguyen TP, Kikuchi M, Vu TQ, et al. Protective and enhancing HLA alleles, HLADRB1*0901 and HLA-A*24, for severe forms of dengue virus infection, dengue hemorrhagic fever and dengue shock syndrome. PLoS Neglected Tropical Diseases 2008; 2: e304.

14. Appanna R, Ponnampalavanar S, Lum Chai See L and Sekaran SD. Susceptible and protective HLA class 1 alleles against dengue fever and dengue hemorrhagic fever patients in a Malaysian population. PLoS One 2010; 5: e13029. 
15. Martina BE, Koraka $\mathrm{P}$ and Osterhaus $\mathrm{AD}$. Dengue virus pathogenesis: an integrated view. Clinical Microbiology Reviews 2009; 22: 564-81.

16. Falcon-Lezama JA, Ramos C, Zuniga J, et al. HLA class I and II polymorphisms in Mexican Mestizo patients with dengue fever. ActaTropica2009: 193-7.

17. Stephens HA, Klaythong R, Sirikong M, et al. HLA-A and -B allele associations with secondary dengue virus infections correlate with disease severity and the infecting viral serotype in ethnic Thais. Tissue Antigens 2002; 60: 309-18.

18. Loke H, Bethell D, Phuong CX, et al. Susceptibility to dengue hemorrhagic fever in Vietnam: evidence of an association with variation in the vitamin $\mathrm{d}$ receptor and $\mathrm{Fc}$ gamma receptor IIa genes. American Journal of Tropical Medicine \& Hygiene2002; 67: 102-6.

19. Chen RF, Wang L, Cheng JT, et al. Combination of CTLA-4 and TGFbetal gene polymorphisms associated with dengue hemorrhagic fever and virus load in a dengue-2 outbreak. Clinical Immunology 2009;131:404-9.

20. Malavige GN, Rostron, T., Rohanachandra, L.T., Jayaratne, S.D., Fernando, N., De Silva A.D., Liyanage, M., Ogg, G.S. HLA class I and class II associations in dengue viral infections in a Sri Lankan population. PLoS One In press.

21. Chau TN, Quyen NT, Thuy TT, et al. Dengue in Vietnamese infants--results of infection-enhancement assays correlate with age-related disease epidemiology, and cellular immune responses correlate with disease severity. Journal of Infectious Diseases 2008; 198: 516-24.

22. Thomas L, Verlaeten O, Cabie A, et al. Influence of the dengue serotype, previous dengue infection, and plasma viral load on clinical presentation and outcome during a dengue-2 and dengue-4 co-epidemic. American Journal of Tropical Medicine \& Hygiene 2008; 78: 990-8.

23. Wang WK, Chao DY, Kao CL, et al. High levels of plasma dengue viral load during defervescence in patients with dengue hemorrhagic fever: implications for pathogenesis. Virology 2003; 305: 330-8.

24. Guilarde AO, Turchi MD, Siqueira JB, Jr., et al. Dengue and dengue hemorrhagic fever among adults: clinical outcomes related to viremia, serotypes, and antibody response. Journal of Infectious Diseases 2008; 197: 817-24.

25. Mathew A, Kurane I, Green S, et al. Predominance of HLA-restricted cytotoxic Tlymphocyte responses to serotype-cross-reactive epitopes on nonstructural proteins following natural secondary dengue virus infection. Journal of Virology 1998; 72: 3999-4004.

26. Beaumier CM, Mathew A, Bashyam HS and Rothman AL. Cross-reactive memory $\mathrm{CD} 8(+) \mathrm{T}$ cells alter the immune response to heterologous secondary dengue virus infections in mice in a sequence-specific manner. Journal of Infectious Diseases 2008; 197: 608-17.

27. Simmons CP, Dong T, Chau NV, et al. Early T-cell responses to dengue virus epitopes in Vietnamese adults with secondary dengue virus infections. Journal of Virology 2005; 79: 5665-75.

28. Durbin AP, Vargas MJ, Wanionek K, et al. Phenotyping of peripheral blood mononuclear cells during acute dengue illness demonstrates infection and increased activation of monocytes in severe cases compared to classic dengue fever. Virology 2008; 376: 429-35.

29. Dong T, Moran E, Vinh Chau N, et al. High pro-inflammatory cytokine secretion and loss of high avidity cross-reactive cytotoxic T-cells during the course of secondary dengue virus infection. PLoS One 2007; 2:e1192. 
30. Mongkolsapaya J, Duangchinda T, Dejnirattisai $\mathrm{W}$, et al. $\mathrm{T}$ cell responses in dengue hemorrhagic fever: are cross-reactive T cells suboptimal? Journal of Immunology 2006; 176: 3821-9.

31. Appanna R, Huat TL, See LL, Tan PL, Vadivelu J and Devi S. Cross-reactive T-cell responses to the nonstructural regions of dengue viruses among dengue fever and dengue hemorrhagic fever patients in Malaysia. Clinical and Vaccine Immunology 2007; 14: 969-77.

32. Imrie A, Meeks J, Gurary A, et al. Differential functional avidity of dengue virusspecific T-cell clones for variant peptides representing heterologous and previously encountered serotypes. Journal of Virology 2007; 81: 10081-91.

33. Ong A, Sandar M, Chen MI and Sin LY. Fatal dengue hemorrhagic fever in adults during a dengue epidemic in Singapore. International Journal of InfectiousDiseases 2007; 11: 263-7.

34. Malavige GN, Velathanthiri VG, Wijewickrama ES, et al. Patterns of disease among adults hospitalized with dengue infections. QJM 2006; 99: 299-305.

35. Waduge R, Malavige GN, Pradeepan $\mathrm{M}$, Wijeyaratne $\mathrm{CN}$, Fernando $\mathrm{S}$ and Seneviratne SL. Dengue infections during pregnancy: a case series from Sri Lanka and review of the literature. Journal of Clinical Virology 2006; 37: 27-33.

36. Malavige GNC, L.; Peelawatta, M.; Fernando, N.; Jayaratne, S.D.; Ogg, G.S. Dengue virus NS3 protein specific $\mathrm{T}$ cell responses in healthy individuals with past dengue infection. British Society of Immunology, Annual Congress. Liverpool,UK: British Society of Immunology, 2010.

37. Luhn K, Simmons CP, Moran E, et al. Increased frequencies of CD4+ CD25(high) regulatory $\mathrm{T}$ cells in acute dengue infection. Journal of Experimental Medicine 2007; 204: 979-85.

38. Chen LC, Lei HY, Liu CC, et al. Correlation of serum levels of macrophage migration inhibitory factor with disease severity and clinical outcome in dengue patients. American Journal of Tropical Medicine \& Hygiene 2006; 74: 142-7. 